ARTICLE

DOI: $10.1038 / s 41467-018-06411-x$

\title{
Genome mining of cyclodipeptide synthases unravels unusual tRNA-dependent diketopiperazine-terpene biosynthetic machinery
}

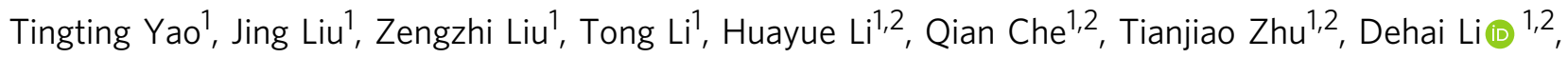
Qianqun $\mathrm{Gu}^{1,2} \&$ Wenli Li (1) ${ }^{1,2}$

Cyclodipeptide synthases (CDPSs) can catalyze the formation of two successive peptide bonds by hijacking aminoacyl-tRNAs from the ribosomal machinery resulting in diketopiperazines (DKPs). Here, three CDPS-containing loci (dmt1-3) are discovered by genome mining and comparative genome analysis of Streptomyces strains. Among them, CDPS DmtB1, encoded by the gene of $d m+1$ locus, can synthesize cyclo(L-Trp-L-Xaa) (with Xaa being Val, Pro, Leu, lle, or Ala). Systematic mutagenesis experiments demonstrate the importance of the residues constituting substrate-binding pocket $\mathrm{P} 1$ for the incorporation of the second aatRNA in DmtB1. Characterization of dmt1-3 unravels that CDPS-dependent machinery is involved in CDPS-synthesized DKP formation followed by tailoring steps of prenylation and cyclization to afford terpenylated DKP compounds drimentines. A phytoene-synthase-like family prenyltransferase (DmtC1) and a membrane terpene cyclase (DmtA1) are required for drimentines biosynthesis. These results set the foundation for further increasing the natural diversity of complex DKP derivatives.

\footnotetext{
${ }^{1}$ Key Laboratory of Marine Drugs, Ministry of Education of China, School of Medicine and Pharmacy, Ocean University of China, 266003 Qingdao, China. ${ }^{2}$ Laboratory for Marine Drugs and Bioproducts of Qingdao National Laboratory for Marine Science and Technology, 266003 Qingdao, China. These authors contributed equally: Tingting Yao, Jing Liu. Correspondence and requests for materials should be addressed to W.L. (email: liwenli@ouc.edu.cn)
} 
$\mathrm{N}$ atural products featuring 2,5-diketopiperazine (DKP) scaffolds are structurally diverse and widely produced by bacteria, fungi, plants, and animals ${ }^{1}$. The core DKP scaffold makes these privileged structures resistant to proteolysis and capable of crossing the intestinal barrier and blood-brain barrier (BBB) ${ }^{2}$. Various compounds based on naturally occurring DKPs have recently gained much attention and are currently being investigated as potential anticancer drugs, antibiotics, and anti-inflammatory agents, such as plinabulin (NPI-2358) ${ }^{3}$, phenylahistin ${ }^{4}$, and bicyclomycin ${ }^{5}$.

The DKP-ring system is formed through double condensation of two a-amino acids via basically two mechanisms: nonribosomal peptide synthetase (NRPS) ${ }^{6}$ and cyclodipeptide synthase (CDPS) ${ }^{7,8}$. Unlike NRPSs, which use free amino acids by activating them through adenylation, CDPSs hijack aminoacyltRNAs (aa-tRNAs) from their primary use in the translation process $^{9}$. Functionally characterized CDPSs are essentially from three bacteria phyla (Actinobacteria, Firmicutes, and Proteobacteria) ${ }^{10,11}$. Albeit displaying low sequence identity (mostly less than 30\%), they fall into two main phylogenetically distinct subfamilies, namely $\mathrm{NYH}$ and $\mathrm{XYP}^{10-12}$. CDPSs characterized to date are able to generate different DKPs, consisting of 18 of the 20 proteinogenic amino acids ${ }^{10,11}$ (with the exception of Lys and Asp) and 26 noncanonical amino acids ${ }^{13}$. The crystallographic structures, as well as mutagenesis studies revealed that the two aatRNAs bind at different substrate-binding sites: pocket P1 and pocket P2. The specificity of the first aa-tRNA depends on its aminoacyl moiety, conversely that of the second aa-tRNA lies on both the aminoacyl moiety and its tRNA sequence ${ }^{14}$. Based on multiple sequence alignments of functionally characterized CDPSs, Jacques et al. proposed the sequence signatures of P1 and $\mathrm{P} 2$ associated with the formation of DKP product ${ }^{11}$. However, the impact of mutagenesis of the residues in P2 along with the interaction between $\mathrm{P} 1$ and $\mathrm{P} 2$ is still obscure.

Attachment of various side chains, such as methyl, prenyl, and hydroxyl, enables DKPs to interact with an array of biological targets $^{1}$. As a matter of fact, CDPSs generally occur associated with tailoring enzymes ${ }^{15}$. Nonetheless only four CDPS-dependent pathways have been fully understood so far, including albonoursin $^{7,16}$, nocazines ${ }^{17,18}$, pulcherriminic acid $^{19}$, and bicyclomycin ${ }^{20,21}$. Compared to the canonic NRPS pathways, CDPS pathways are still underexplored. The type of CDPSsynthesized DKPs combined with modifications would greatly expand the chemical diversity of DKP-based compounds.

In this study, genome mining of marine Streptomyces strains reveals a three-gene CDPS-containing locus (named dmt1) in Streptomyces youssoufiensis OUC6819 (formerly named Streptomyces sp. CHQ-64 ${ }^{22}$ ). In silico analysis of the Streptomyces genomes in GenBank uncovers another two CDPS-containing loci homologous to dmt1 from Streptomyces sp. NRRL F-5123 (named $d m t 2$ ) and Streptomyces aidingensis CGMCC 4.5739 (named $d m t 3$ ). Phylogenetic analysis reveals that these three CDPSs (named DmtB1-3) belong to the cWX-forming group of the NYH subfamily. Functional characterization demonstrates that DmtB1 is a CDPS synthesizing cyclo(L-Trp-L-Xaa) (X = Val, Pro, Leu, Ile, or Ala) with cWV being the major product. The underlying molecular basis for substrate specificity of DmtB1 is then probed via extensive mutagenesis of the amino acid residues in the two binding pockets. Furthermore, these three CDPSdependent biosynthetic loci are characterized and revealed a CDPS-dependent pathway whereby the DKP product is prenylated and cyclized by tailoring enzymes to afford terpenylated diketopiperazine compounds, dubbed drimentines (DMTs, Fig. 1). Notably, a phytoene-synthase-like (PSL) family<smiles>CC1NC(=O)C(C)(Cc2c[nH]c3ccccc23)NC1=O</smiles>

1: cyclo (L-Trp-L-Ala)

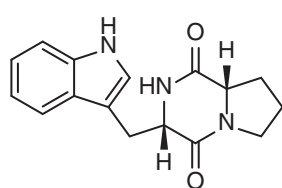

2: cyclo (L-Trp-L-Pro)

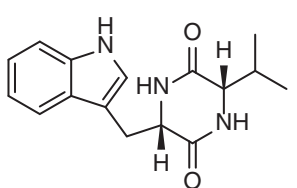

3: $\operatorname{cyclo~(L-Trp-L-Val)~}$

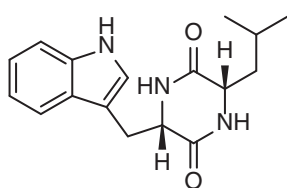

4: cyclo (L-Trp-L-Leu)

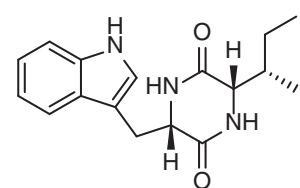

5: cyclo (L-Trp-L-lle) b

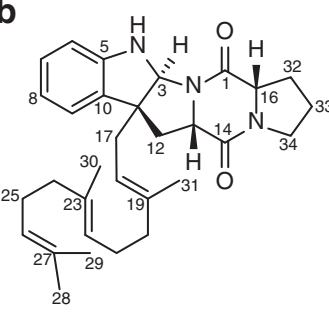

6: pre-DMT C

C<smiles>[R]N1C(=O)[C@]2(C)C[C@]3(C[C@H]4C(=C)CC[C@@]5(C)C(C)(C)CCC[C@@]45C)c4ccccc4N[C@@H]3N2C(=O)C1(C(C)C)C(C)C</smiles>

9: $\mathbf{R}=\mathrm{H}, \mathrm{DMT} \mathbf{G}$ 12: $\mathrm{R}=\mathrm{CH}_{3}$, DMT F

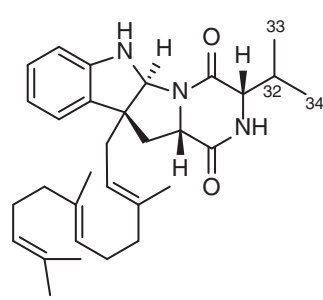

7: pre-DMT G

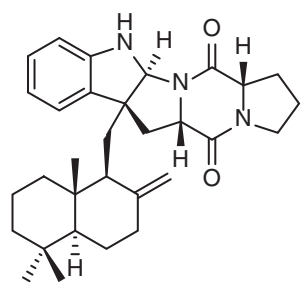

10: DMT C

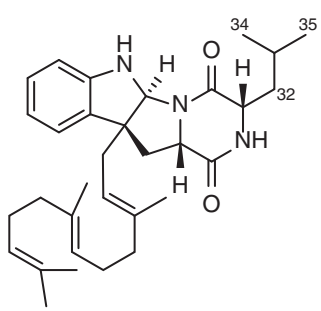

8: pre-DMT A

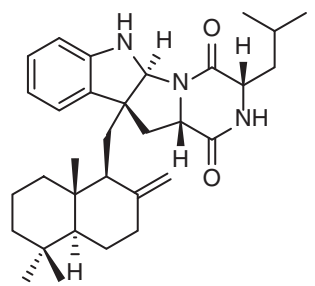

11: DMT A

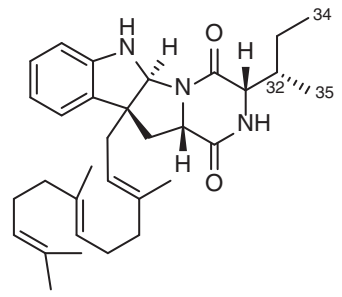

13: pre-DMT J

Fig. 1 Chemical structures of compounds 1-13. a Diketopiperazines (DKPs) synthesized by DmtB1 (1-5); b pre-drimentines (pre-DMTs, 6-8 and 13); c drimentines (DMTs, 9-12) 


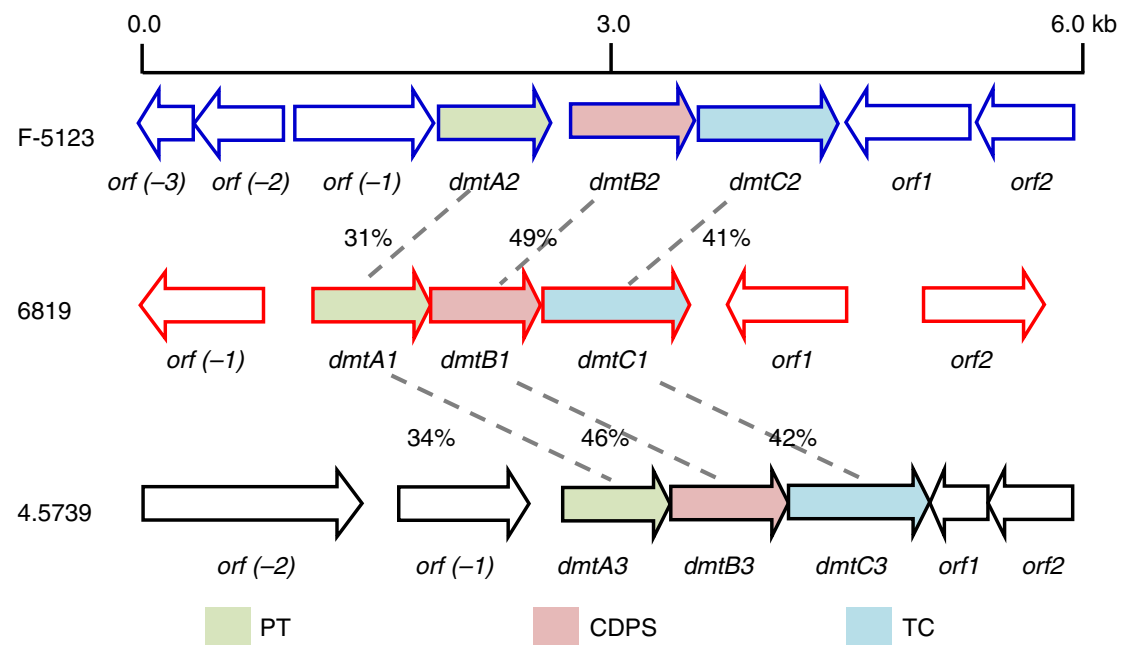

Fig. 2 Comparison of the three CDPS-containing loci dmt1-3. The dmt1-3 are from S. youssoufiensis OUC6819, Streptomyces sp. NRRL F-5123 and S. aidingensis CGMCC 4.5739, respectively

Table 1 Predicted functions of the dmtA1-C1

\begin{tabular}{lllll} 
Protein & Size (aa) & Proposed function & Homologs & \\
\cline { 4 - 5 } & & & Protein/organism & Accession no. (Identity/similarity \%) \\
\hline DmtA1 & 250 & Hypothetical protein & XiaE/Streptomyces sp. HKI0576 & CCH63731.1 (30/42) \\
DmtB1 & 233 & Cyclodipeptide synthase, CDPS & Amir_4627/Actinosynnema mirum DSM 43827 & ACU38460.1 (37/52) \\
DmtC1 & 311 & Phytoene synthase & NzsG/Streptomyces sp.MA37 & ALL53320.1 (21/33)
\end{tabular}

prenyltransferase (PT) and a membrane terpene cyclase (TC) are identified. Thus, this study expands our understanding of CDPSs, as well as CDPS-dependent biosynthetic pathways, setting the stage to further increase the natural diversity of complex DKP derivatives using combinatorial approaches.

\section{Results}

Genome mining reveals three homologous CDPS-containing loci. Genome mining of marine-derived $S$. youssoufiensis OUC6819 revealed a putative three-gene operon $d m t 1$, containing a CDPS gene dmtB1 (Fig. 2). An additional two homologous loci were then identified in Streptomyces sp. NRRL F-5123 (dmt2) and $S$. aidingensis CGMCC 4.5739 (dmt3) following in silico analysis of Streptomyces genomes available on GenBank (Fig. 2). DmtB1 displayed 37\% identity to Amir_4627 (ACU38460.1) (Table 1), $49 \%$ to DmtB2, and $46 \%$ to DmtB3 (Fig. 2). Phylogenetic analysis revealed that DmtB1-3 belong to the NYH subfamily and are clustered with cWX-synthesizing CDPSs (Supplementary Fig. 1). Notably, DmtB1 bears a Gln203 (numbering after $\mathrm{AlbC}^{14}$ ) instead of His203, which is strictly conserved among the NYH subfamily ${ }^{11}$. To investigate their encoding DKP product(s), the dmtB1-3 genes were cloned into pET-28a and subsequently introduced into $E$. coli BL21(DE3). High-performance liquid chromatography (HPLC) analysis of the culture supernatants showed that five DKP compounds 1-5 were produced by E. coli cells containing dmtB1 (Fig. 3). These compounds were identified as cyclo(L-Trp-L-Ala) (1), cyclo(L-Trp-L-Pro) (2), cyclo(L-Trp-LVal) (3), cyclo(L-Trp-L-Leu) (4), and cyclo(L-Trp-L-Ile) (5) based on their retention times (Fig. 3) and their high-resolution mass spectra (HR-MS, Supplementary Figs. 3-7) in comparison to those of authentic standards. Conversely, expression of $d m t B 2$

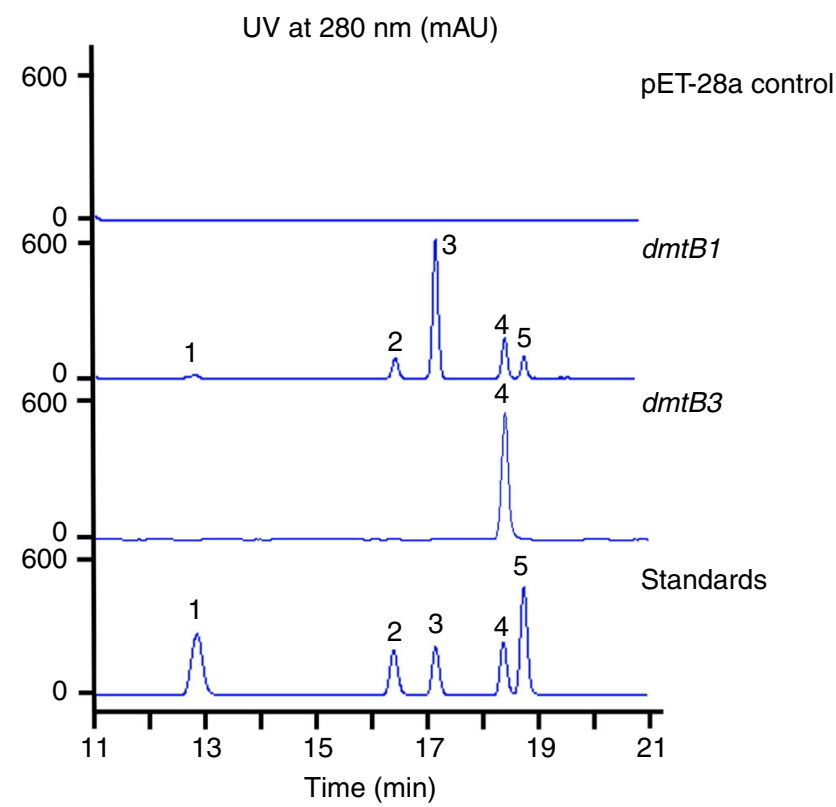

Fig. 3 HPLC traces of culture supernatants of $E$. coli cells expressing DmtBs

yielded 2 plus a minor amount of 4 (Supplementary Fig. 2), consistent with the recently published result ${ }^{23}$, and DmtB3 only synthesized 4 (Fig. 3). These results highlighted the substrate promiscuity of DmtB1 regarding to the second amino acid residue incorporated into DKPs. In contrast, DmtB2 and DmtB3 exhibit a relatively stringent specificity. 
a
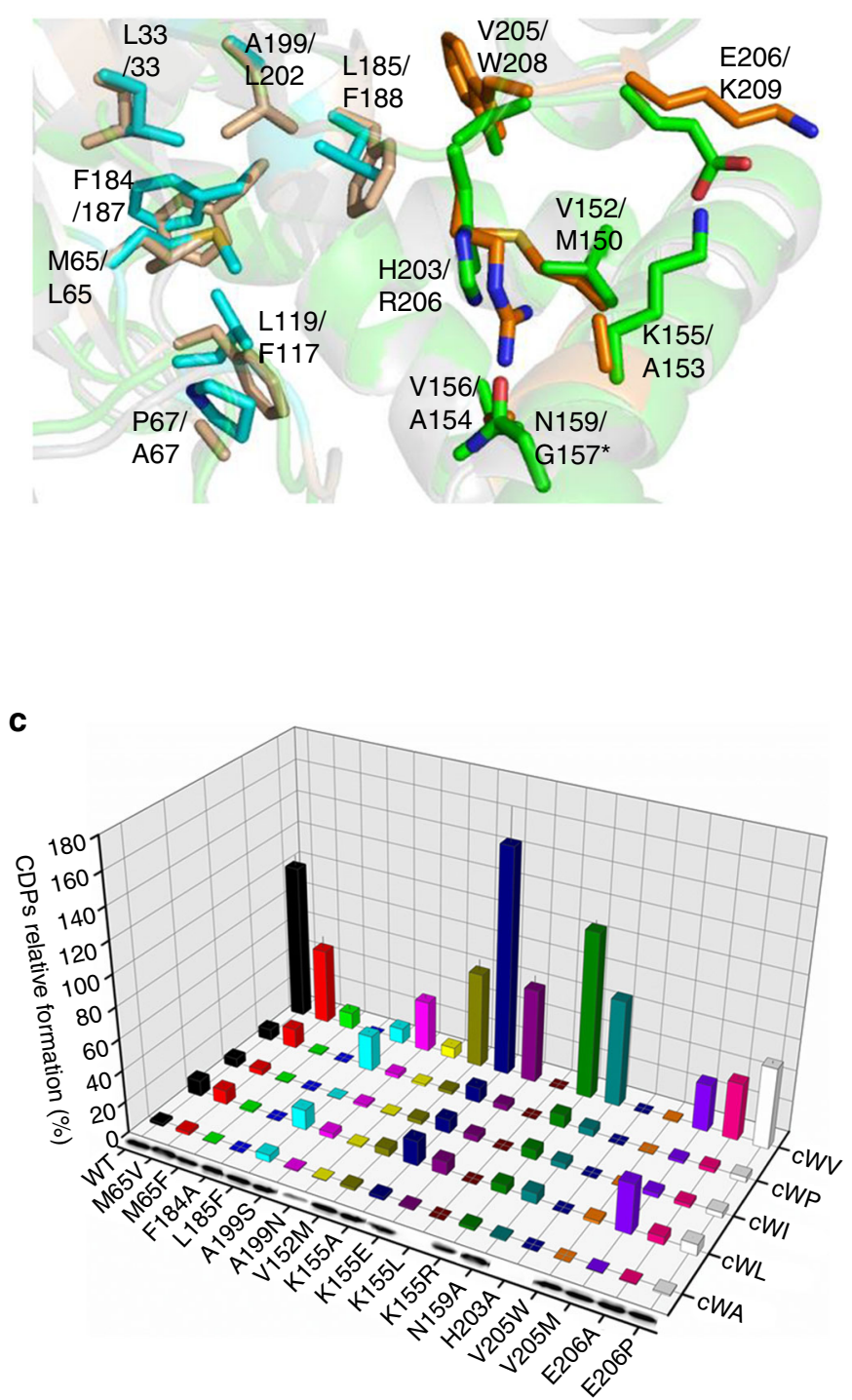

b

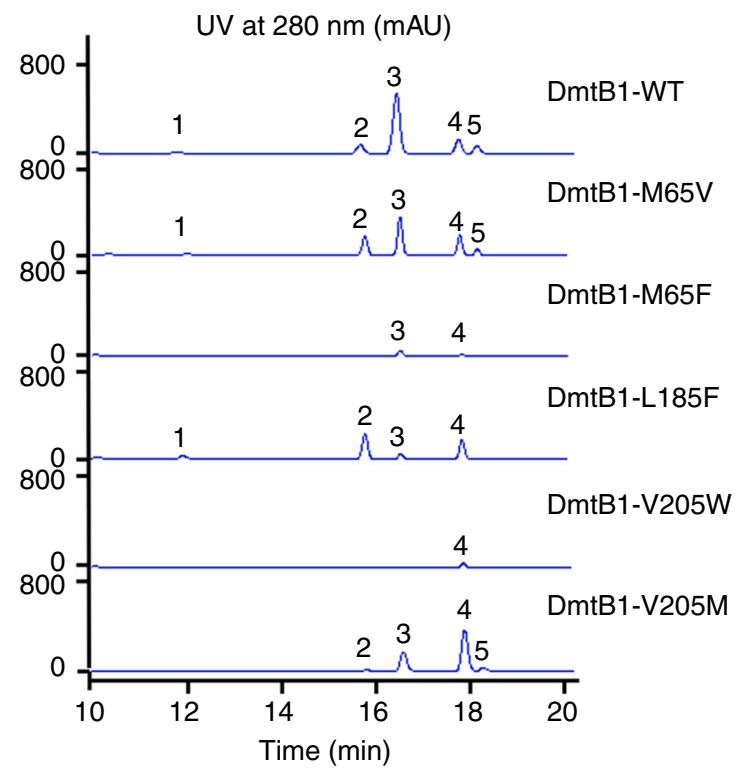

d

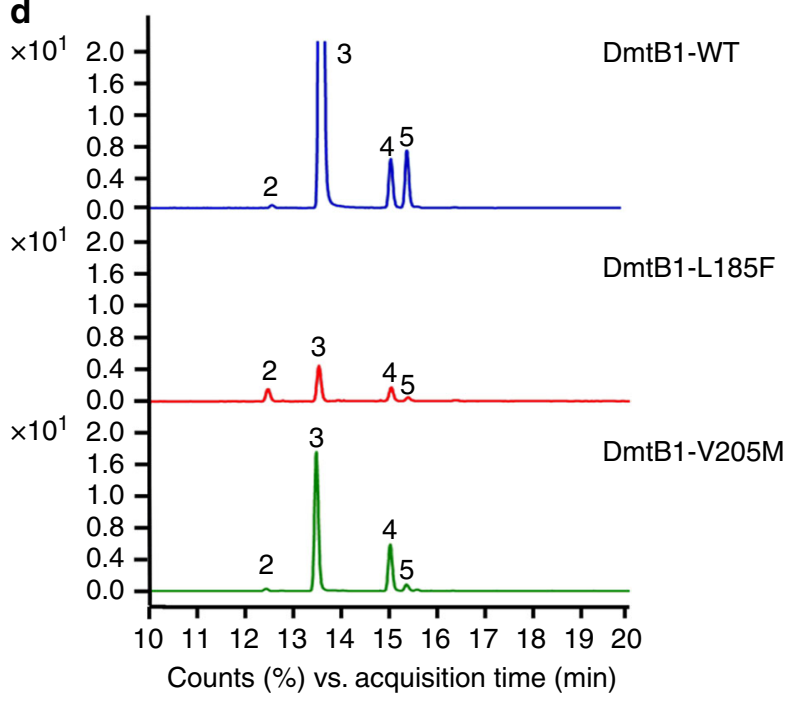

Fig. 4 Mutagenesis of the residues located in the binding pockets of DmtB1. a Superposition of the binding pockets in the DmtB1 model with those in the crystal structure of YvmC. The P1 and P2 of the DmtB1 model are shown in cyan and green, respectively; the P1 and P2 of YvmC (PDB id: $30 Q \mathrm{~J}$ ) are shown in wheat and brown, respectively. b HPLC traces of the products formation by DmtB1 and its variants (also see Supplementary Fig. 10). c Histogram of the relative formation of various DKPs synthesized by DmtB1 and its variants after $20 \mathrm{hrs}$ of expression in $\mathrm{E}$. coli at $16^{\circ} \mathrm{C}$. The corresponding western blots indicating amounts of proteins are also shown. Error bars represent \pm s.d. of three independent experiments. $\mathbf{d}$ Total ion chromatogram in multiple reaction monitoring (MRM) mode for compounds $\mathbf{2 - 5}$ resulting from in vitro assays of DmtB1 and its variants (L185F and V205M). The relative titers of compounds were compared based on the peak area of MS $^{2}$ fragment 130.11 from each molecular ion

Probing the molecular basis for DmtB1 substrate specificity. With the three orthologous CDPSs in hand, we probed the underlying molecular basis for the substrate specificity. Firstly, the binding pockets $\mathrm{P} 1$ and P2, accommodating the aminoacyl moieties of the first and second aa-tRNAs, were predicted by multiple sequence alignment (Supplementary Fig. 8, Supplementary Data 2). As shown in Supplementary Data 2, although $\mathrm{P} 1$ and P2 are independent, the residues constituting the P1 pockets of DmtB1-3 are not well conserved with those of the cWW-synthesizing CDPSs. DmtB1-3 harbor a L186 (AlbC numbering) instead of Phe, and contain a hydrophobic residue at position 200 (Ala or Leu) other than hydrophilic residues present in cWW-synthesizing CDPSs. The residues constituting the P2 pockets of DmtB1-3 display a striking difference to those of
cWW-synthesizing CDPSs at position 207 by replacing Pro with an acidic amino acid Glu (in DmtB1 and DmtB3) or Asp (in DmtB2). Except V156, other residues in P2 are dissimilar among the aligned CDPSs.

A structural model of DmtB1 was then generated using the protein threading and fold recognition server I-TASSER ${ }^{24}$ to assist understanding the underlying molecular basis of substrate specificity (Supplementary Fig. 9). As shown in Fig. 4a, the binding pockets $\mathrm{P} 1$ and $\mathrm{P} 2$ of the structural model of DmtB1 superimposed well with those of $\mathrm{YvmC}^{25}$ (PDB id: 3OQJ). To test the key residue(s) for substrate specificity, we performed a series of mutagenesis of selected residues in both pocket P1 (M65, F184, L185 and A199) and pocket P2 (V152, K155, N159, H203, V205, and E206). The resulting variants were 


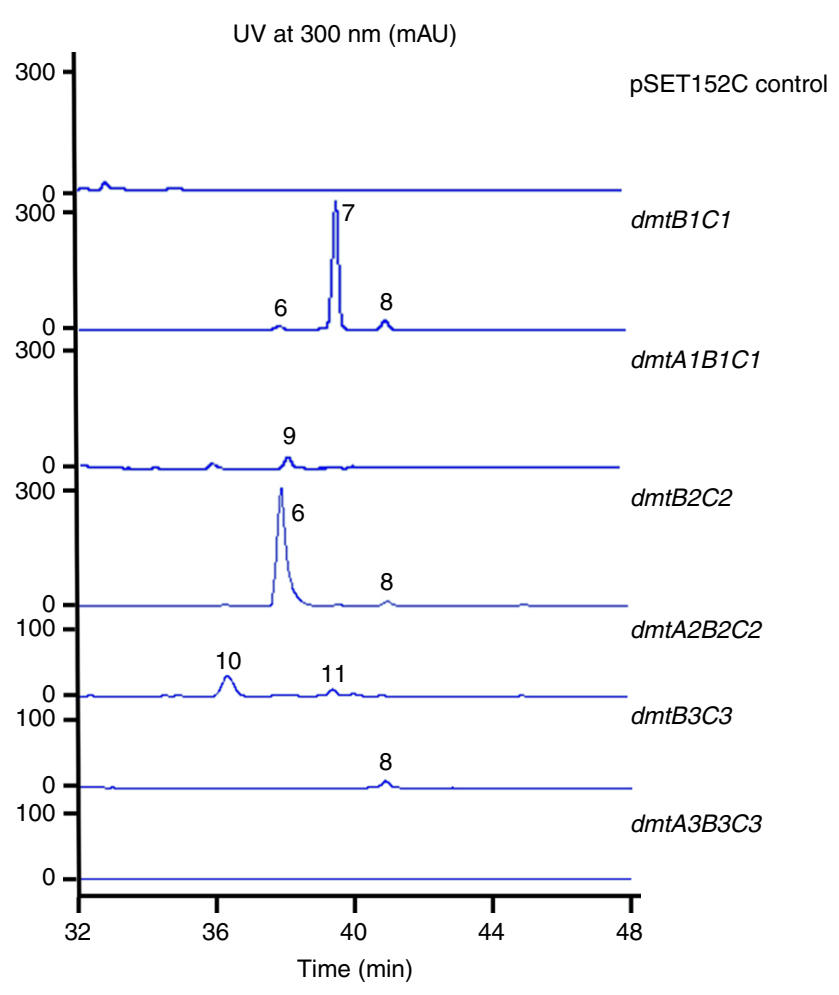

Fig. 5 Heterologous expression of the dmt1-3 loci in S. coelicolor M1146

introduced into E. coli, and in vivo synthesis of DKPs, as well as recombinant protein expression levels were compared to those of the wild-type DmtB1 (Fig. 4, Supplementary Fig. 10).

For the residues in the pocket $\mathrm{P} 1$, the variant $\mathrm{M} 65 \mathrm{~V}$ synthesized increased amount of cyclo(L-Trp-L-Pro) (2, by $\sim 1.7$ fold) but decreased amount of $c y c l o(\mathrm{~L}-\mathrm{Trp}-\mathrm{L}-\mathrm{Val})(3$, by $\sim 2$-fold $)$ and $c y c l o(\mathrm{~L}-\mathrm{Trp}$-L-Ile) $(5$, by $\sim 2$-fold), and conversely, the variant M65F was poorly active despite being abundantly produced (Fig. 4b, c). Substitution of L185 with Phe led to significant change of the production of these five DKPs: inability to synthesize 5 , strong defect to form 3 (decreased by $\sim 10$-fold), and yield improvement of $\mathbf{1}$ and $\mathbf{2}$ by $\sim 2.4$ and 3.1-fold, respectively, and thus the major product switched from $\mathbf{3}$ to $\mathbf{2}$. When V205 in the pocket P2 was mutated into Trp or Met, the variant $\mathrm{V} 205 \mathrm{~W}$ only synthesized a very low amount of $\mathbf{4}$ and the major product of the variant V205M shifted from $\mathbf{3}$ to $\mathbf{4}$ with increased amount by about 2.3 -fold and simultaneously decreased $\mathbf{2 , 3}$, and $\mathbf{5}$ or abolished $\mathbf{1}$ production (Fig. $4 \mathrm{~b}, \mathrm{c}$ ). These results clearly indicate that substitution of residues constituting either P1 or $\mathrm{P} 2$ pocket could affect the nature of the second amino acid incorporated.

Considering the in vivo results are dependent on cellular availability of specific aa-tRNA substrates, we further performed in vitro assays for DmtB1 and its variants, L185F and V205M, to closely control the concentrations of the aa-tRNA substrates. As shown in Fig. 4d, in comparison to the wild-type DmtB1, the 3forming ability of both variants was significantly decreased by $\sim 43$-fold (L185F) and 6-fold (V205M), but 3 still served as the most abundant product; conversely, the $\mathbf{2}$-forming ability of L185F was improved by 2.5 -fold, and no obvious change was observed for the 4-forming ability of V205M. Although the major product synthesized by L185F and V205M in vitro is different from that detected in vivo (Fig. $4 \mathrm{~b}$ ), the changes in the formation ratios of $\mathbf{2}$ vs. 3 and $\mathbf{4}$ vs. 3 between the wild-type DmtB1 and the variants were consistent in vivo and in vitro (Supplementary
Table 7). Thus, the importance of L185 and V205 for substrate specificity could be safely established.

Furthermore, based on the mutagenesis results for DmtB1, the equivalent residues in DmtB2 (V74 and L194 in pocket P1, and I214 in pocket P2, Supplementary Data 2) were mutated, and the result demonstrated L194 (corresponding to L185 in DmtB1) might serve as an important substrate specificity determinant in DmtB2 in vivo (Supplementary Fig. 11).

Inspection of the genetic contexts of dmtB1-3. As CDPSs generally occur associated with tailoring enzymes, we next inspected the genetic contexts of the dmtB1-3, disclosing the presence of conserved membrane protein DmtA1-3 and phytoene synthase DmtC1-3 (Fig. 2). DmtA1 displayed 30\% identity to the membrane terpene cyclase XiaE (CCH63731.1) (Table 1) and showed identities of $31 \%$ and $34 \%$ to DmtA2 and DmtA3, respectively (Fig. 2). DmtC1 displayed $21 \%$ identity to PSL prenyltransferase NzsG (ALL53320.1) (Table 1), and showed identities of $41 \%$ and $42 \%$ to DmtC2 and DmtC3, respectively (Fig. 2). Hence the dmt1-3 loci were predicted to encode DKPs with a terpene moiety. Further inspection into the genetic neighborhood of the dmt1-3 loci showed: (i) genes surrounding the $d m t 1$ locus displayed no homology to those of the $d m t 2 / 3$ loci; (ii) homologs of the two subunits of cyclic dipeptide oxidase (CDO) were found neighboring to the $d m+2 / 3$ loci, suggesting potential $\alpha, \beta$-dehydrogenation(s) would happen on the DKP moiety; (iii) the adjacent regions of the $d m t 2 / 3$ loci also harbor putative methyltransferase gene(s), sharing low homology to each other and to characterized methyltransferases from CDPS-containing loci ${ }^{18,26}$ (Supplementary Fig. 12).

To identify and characterize the metabolic products of these three assemble lines, a series of expression plasmids were constructed. $d m t B C$ and $d m t A B C$ from each cluster were cloned under the control of the Streptomyces constitutive promoter $\mathrm{P}_{\text {gapdh }}$, resulting in pWLI615-620. These constructs were then introduced into the superhost Streptomyces coelicolor M1146. As shown in Fig. 5, expression of $d m t B 1 C 1$ led to accumulation of three compounds 6-8, which presumably derive from compounds 2-4. By further introducing $d m t A 1$, a single compound 9 was produced in a low titer, which was identified as drimentine $G$ (DMT G, Fig. 1, Supplementary Figs. 13-15) harboring a cWV derivative moiety and was previously isolated from the fermentation broths of S. youssoufiensis OUC681922

For the expression of the dmt2 locus, M1146/pWLI617 (expressing $d m t B 2 C 2$ ) produced compounds $\mathbf{6}$ and $\mathbf{8}$, and $d m t A 2 B 2 C 2$ accumulated compounds $\mathbf{1 0}$ and $\mathbf{1 1}$ (Fig. 5), which were identified to be DMT C and DMT A, respectively, based on comparison with retention times of authentic standards (Supplementary Fig. 13), as well as HR-MS and ${ }^{1} \mathrm{H}$ NMR data (Supplementary Figs. 16-19). When we expressed $d m t B 3 C 3$, only a small amount of compound $\mathbf{8}$ was produced, and no product was observed after further introduction of $d m t A 3$ (Fig. 5). Taken together these results imply that $\mathrm{DmtCl}$ catalyses the condensation of a farnesyl diphosphate (FPP) moiety to $c y c l o(\mathrm{~L}-\mathrm{Trp}-\mathrm{L}-\mathrm{Val})$ (3). The resulting compound 7 is then proposed to be cyclized by DmtA1 to yield DMT G (9). Similarly, cyclo(L-Trp-L-Pro) (2) and cyclo(L-Trp-L-Leu) (4) assembled by DmtB2 are converted into compounds 6 and 8 by DmtC2, before being cyclized by DmtA2 to yield DMT C (10) and DMT A (11).

In vivo characterization of the $\boldsymbol{d} \boldsymbol{m} \boldsymbol{t} \mathbf{1}$ locus. To establish the function of the dmt1 locus in the native micro-organism, gene inactivation was then carried out and resulted in the following three mutant strains $S$. youssoufiensis OUC6819 $\triangle d m t A 1, \Delta d m t B 1$ and $\triangle d m t C 1$ (Supplementary Figs. 20-22). HPLC analysis of the 
culture extracts from these mutants revealed that production of DMT G (9) and DMT F (12), both naturally produced by the wild-type strain, was abolished in every mutant strain (Fig. 6). These results demonstrated that DmtA1, DmtB1 and DmtC1 are all essential for DMT biosynthesis in S. youssoufiensis OUC6819. Simultaneously, accumulation of compounds 6-8 was observed in the $\Delta d m t A 1$ culture extract (Fig. 6) with an additional compound 13 in comparison to those in S. coelicolor M1146 expressing $d m t B 1 C 1$ (Fig. 5). The ratios among 6-8 were analogous in both strains, with 7 being the most abundant intermediate. Genetic complementation of the three mutants by overexpression of $d m t A 1, d m t B 1$, and $d m t C 1$ were also performed. The results showed that compounds $\mathbf{9}$ and $\mathbf{1 2}$ were overproduced when $d m t A 1, d m t B 1$, or $d m t C 1$ were overexpressed in the wild-type strain background (Supplementary Figs. 23-25). The $\Delta d m t B 1$ (Supplementary Fig. 24) and $\Delta d m t C 1$ (Supplementary Fig. 25) mutants complemented with $d m t B 1$ and $d m t C 1$, respectively, were able to produce DMTs but in limited amount. Conversely, $\triangle d m t A 1$ strain complemented with $d m t A 1$ accumulated both 9 and $\mathbf{1 2}$ in much higher yield than those in the wild-type strain. This complemented strain still produced compounds $\mathbf{6 - 8 , 1 3}$ as $\Delta d m t A 1$ did (Supplementary Fig. 23). These results further established the essential role of $d m t 1$ in DMT biosynthesis.

Subsequently, large scale fermentation of the $\Delta d m t A 1$ mutant strain followed by chemical isolation was performed, leading to identification of compounds 6-8, 13. HR-ESIMS analysis indicated the molecular formula of 6 as $\mathrm{C}_{31} \mathrm{H}_{41} \mathrm{~N}_{3} \mathrm{O}_{2}(\mathrm{~m} / z$ 488.3272 $[\mathrm{M}+\mathrm{H}]^{+}$, calcd 488.3277, Supplementary Fig. 27), 7 as $\mathrm{C}_{31} \mathrm{H}_{43} \mathrm{~N}_{3} \mathrm{O}_{2}\left(\mathrm{~m} / z 490.3425[\mathrm{M}+\mathrm{H}]^{+}\right.$, calcd 490.3434, Supplementary Fig. 34), 8 as $\mathrm{C}_{32} \mathrm{H}_{45} \mathrm{~N}_{3} \mathrm{O}_{2}\left(m / z 504.3580[\mathrm{M}+\mathrm{H}]^{+}\right.$, calcd 504.3590, Supplementary Fig. 41), and 13 as $\mathrm{C}_{32} \mathrm{H}_{45} \mathrm{~N}_{3} \mathrm{O}_{2}$ $\left(m / z 504.3588[\mathrm{M}+\mathrm{H}]^{+}\right.$, calcd 504.3590, Supplementary Fig. 48), respectively. The structures of these compounds were determined by $1 \mathrm{D}$ and $2 \mathrm{D}$ NMR spectroscopic analysis (Supplementary Table 9, Supplementary Figs. 28-33, 35-40, 42-47, and 49-54). The HMBC correlations from $\mathrm{H}-3$ to $\mathrm{C}-13$ of the DKP ring in each compound suggested the formation of the fused fivemembered ring between the indoline and the DKP ring, which is identical to that in DMTs (Fig. 1). In each ${ }^{13} \mathrm{C}$ spectrum of 6-8,

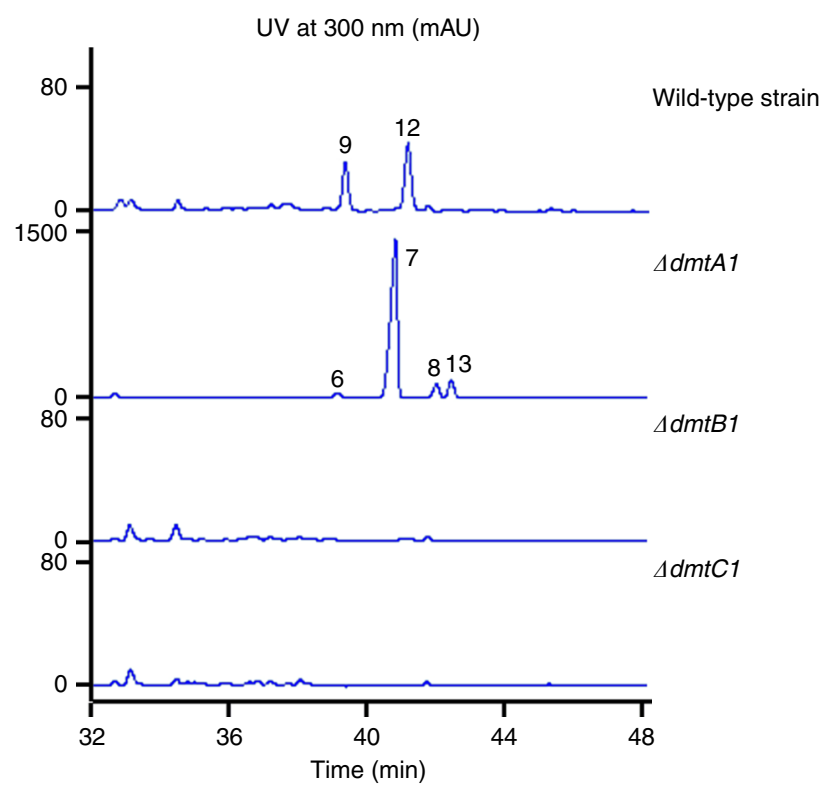

Fig. $6 \mathrm{HPLC}$ traces of the fermentation products from S. youssoufiensis OUC6819 strains
13, we observed fifteen carbon signals, including six olefinic carbons, five methylene carbons and four methyl carbons, which were fully connected by COSY (H-17/H-18, H-20/H-21/H-22, and $\mathrm{H}-24 / \mathrm{H}-25 / \mathrm{H}-26)$ and $\mathrm{HMBC}$ correlations, forming a linear farnesyl chain. The HMBC correlations from $\mathrm{H}-17$ to $\mathrm{C}-3, \mathrm{C}-10$, $\mathrm{C}-11$, and C-12 undoubtedly revealed that the farnesyl chain was attached to the cyclized-Trp residue in each compound. The main structural differences among 6-8, 13 were attributed to the other amino acid residues comprising the DKP skeletons with Trp. According to the ${ }^{1} \mathrm{H}$ spin system of amino acid side chain in COSY, the residues were finally established to be Pro, Val, Leu and Ile for 6-8, 13, respectively. The absolute configurations of C3 and C-11 were determined as $3 S$ and $11 S$, respectively, by comparison of their NMR values with those of DMT G $(\mathbf{1})^{22}$. Since these compounds are encoded by CDPS-dependent pathway, all the amino acid residues were determined to be L-configuration. The configurations of the three double bonds in the farnesyl group were confirmed to be trans by NOE correlations of $\mathrm{H}-17 / \mathrm{H}-31, \mathrm{H}-22 / \mathrm{H}-24$, and $\mathrm{H}-26 / \mathrm{H}-28$. Thus, 68, 13 were finally identified as DMT analogs with linear farnesyl chains, which are proposed the intermediates in DMT biosynthesis. As the potential cyclization product of $\mathbf{1 3}$ would be a putative compound DMT J, 6-8, 13 were named pre-drimentine $\mathrm{C}, \mathrm{G}, \mathrm{A}$, and J, respectively, among which $\mathbf{8}$ has previously been generated by organic synthesis ${ }^{27}$.

DmtC1 is a prenyltransferase belonging to the PSL family. Bioinformatic analysis indicated that DmtC1 has $27-38 \%$ similarity to phytoene/squalene synthases, which catalyze the conversion of geranylgeranyl pyrophosphate (GGPP) or two FPP to yield phytoene and squalene, respectively. The heterologous expression experiments, as well as $d m t C 1$ gene inactivation in $S$. youssoufiensis OUC6819 revealed that DmtC1 is undoubtedly involved in the assembly of the FPP group onto the DKP moieties to generate metabolites that act as substrates for the last enzyme in the pathway, DmtA1. Phylogenetic analysis suggested that DmtC1 is clustered with $\mathrm{NzsG}^{28}$ and squalene/phytoene synthases instead of typical aromatic or indole PTs (Supplementary Fig. 55). To demonstrate the exact function of DmtC1, we overexpressed $d m t C 1$ in E. coli (Supplementary Fig. 56) and carried out biochemical assays. As the $\triangle d m t A 1$ mutant strain accumulated four pre-DMT compounds 6-8 and 13, all the four corresponding DKP compounds 2-5 were tested as substrates for DmtC1, in the presence of FPP. After incubation with DmtC1 for $1 \mathrm{~h}$ at $30^{\circ} \mathrm{C}$, reactions were analyzed by HPLC. As shown in Fig. 7, DmtC1 successfully condensed FPP onto the C-3 position of 2-5 to provide 6-8 and 13 , respectively. The formation of the fused five-membered ring between the indoline and DKP ring (Fig. 7) is possibly a non-enzymatic process or catalyzed by DmtC1 as well. Notably, the relaxed substrate specificity displayed by DmtC1 would enable potential generation of an array of structurally diverse terpenylated Trp-containing DKPs compounds.

DmtA1 initiates cyclization by protonating $\mathrm{C}-\mathrm{C}$ double bond. Heterologous expression and work in S. youssoufiensis OUC6819 also clearly demonstrated the function of DmtA1 as a terpene cyclase. Blastp analysis of DmtA1 revealed it is a membrane protein with seven transmembrane helices (Fig. 8, Supplementary Fig. 57) but did not give any hint on its catalytic activity. The function of the closest homologues $\mathrm{XiaE}^{29} / \mathrm{XiaH}^{30}$ could not be predicted either. In DMT biosynthesis, cyclization is proposed to be triggered by protonation of the $\mathrm{C}-\mathrm{C}$ double bond (between $\mathrm{C} 26$ and $\mathrm{C27}$ ) while protonation of an epoxide ring is involved in xiamycin biosynthesis ${ }^{29,30}$. As initial protonation of an isoprene 



Fig. 7 Biochemical function of DmtC1. a Schematic representation of DmtC1-catalyzed reactions illustrated by using $\mathbf{2}$ as substrate. $\mathbf{b} \mathrm{HPLC}$ traces of DmtC1 biochemical assays

double bond is less favored than that of an oxirane moiety, we further probed this proposed mechanism for DmtA1-catalyzed cyclization. Sequence alignment with DmtA1-3 (227-250 aa) and $\mathrm{XiaE} / \mathrm{XiaH}$, all of which lack the typical aspartate-rich motifs present in TCs, were performed (Supplementary Fig. 58). In addition to the acidic residues E60 and D214 (DmtA1 numbering), conserved in membrane TCs, such as in $\mathrm{Pyr}^{31}{ }^{31}$ where these residues are proposed to catalyze the cyclization reaction, D94 and E133 were found to be uniquely conserved within DmtA1-3 (Supplementary Fig. 58). These acidic residues were therefore mutated to investigate their potential role in catalysis. Unfortunately, $d m t A 1$ was unable to express in E. coli despite many attempts. As an alternative approach, different versions of mutated $d m t A 1$ were constructed and introduced into the $S$. youssoufiensis OUC6819 $\Delta d m t A 1$ mutant where the impact of the amino acid substitutions were determined by assessing the catalytic activity of DmtA1 variants. As shown in Fig. 8, substitution of E60 with Ala or Gln completely abolished genetic complementation of the $\triangle d m t A 1$ mutant, undoubtedly supporting its catalytic role in DmtA1. A very low amount of DMT G $(\mathbf{9})$ could be detected when using the variant $\mathrm{D} 214 \mathrm{~A}$ to complement the $\triangle d m t A 1$ strain. Production of 9 was more significantly restored in the strain complemented with the variant D214N. When D94 was mutated, the variant $\mathrm{D} 94 \mathrm{~A}$ could not complement the $\Delta d m t A 1$ strain while the variant D94N could only form a very limited amount of $\mathbf{9}$, suggesting its critical role in catalysis. Substitution of E133 with Ala also led to defective production of 9 as compared to the complemented strain with the wild-type DmtA1.

Other conserved residues were mutated in DmtA1, including W29, Y33, N56, W59 and Y217. As indicated in Fig. 8c and Supplementary Fig. 59, substitution of W29 and W59 with alanine failed to complement the $\Delta d m t A 1$ strain; no production of 9 was observed, supporting their potential role in stabilizing the favorable conformation required for catalysis. Both Y33A and Y33F resulted in inactive DmtA1, indicating the essential role of its hydroxyl group for cyclization, possibly through positioning and increasing the nucleophilicity of the acidic residues. Conversely, Y217A was poorly active and Y217F displayed improved activity but still much lower than that of the wild-type DmtA1. A previously reported the acidity of Asp could be increased by a nearby $\mathrm{Cys}^{32}$. The C58 residue unique to DmtA1 (Supplementary Fig. 58) and close to E60 was also mutated to Ala but had almost no influence on its activity. Interestingly, substitution of N56, which is also adjacent to E60, with Ala led to poorly active variant (Fig. 8c, Supplementary Fig. 59), suggesting that might increase E60 acidity rather than C58.

\section{Discussion}

With the increasing availability of genomic information, members of the CDPS family have expanded significantly. Our study revealed two NYH subfamily CDPSs DmtB1 and DmtB3. DmtB1 is a cWX-synthesizing CDPS with cWV being the main major product; while DmtB3 only synthesizes $\mathrm{cWL}, \mathrm{DmtB} 2$ produces cWP plus a small amount of cWL. Concerning the different substrate specificity exhibited by DmtB1-3, systematic mutagenesis experiments were carried out to establish which key residues impact on specificity. Thus, the important contribution of residues lining the substrate binding pockets $\mathrm{P} 1$ and $\mathrm{P} 2$ to the incorporation of the second aa-tRNA was established.

Although the first aa-tRNA of DmtB1-3 is the same as that of the other cWW-synthesizing CDPSs, residues lining the binding pocket $\mathrm{P} 1$ were not well conserved, indicating that in addition to the pocket P2, the recognition of the second aa-tRNA might not only depend on the residues in P2 but could also be dependent on key residues related to the pocket P1. Variants mutated in P1 such as DmtB1-V65M, DmtB1-L185F (Fig. 4), as well as DmtB2- 
a
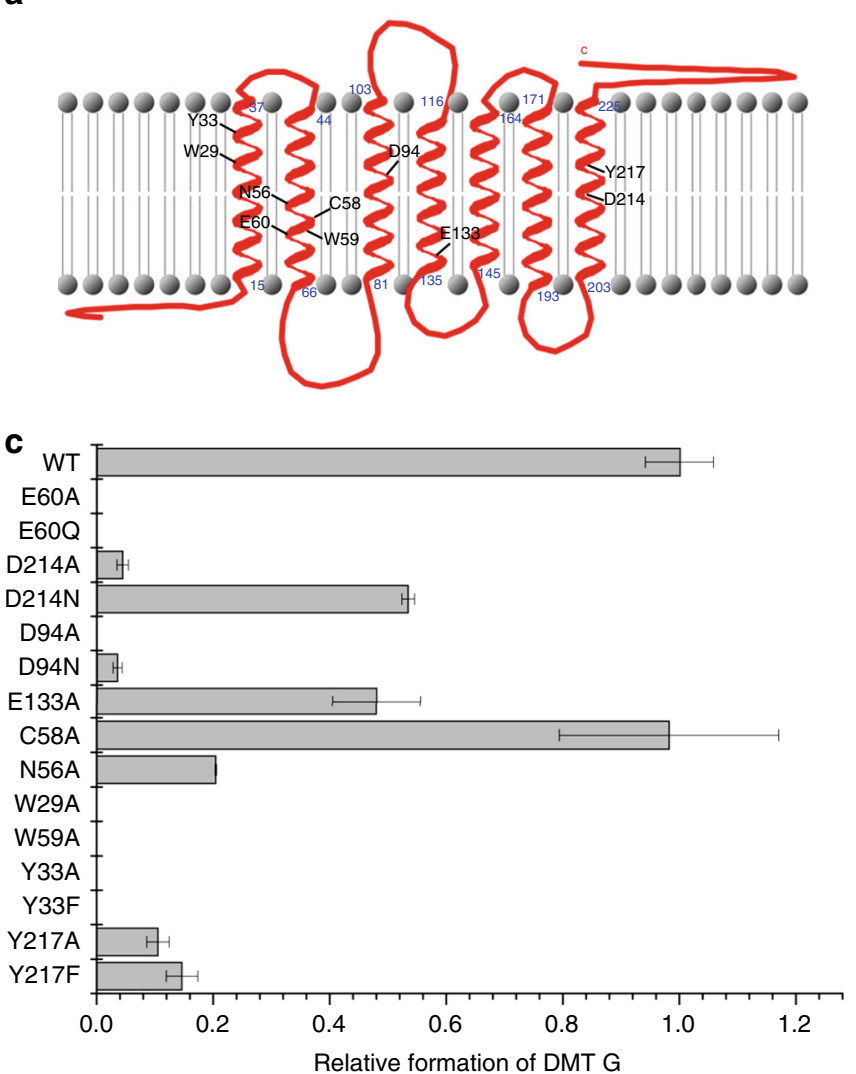

b

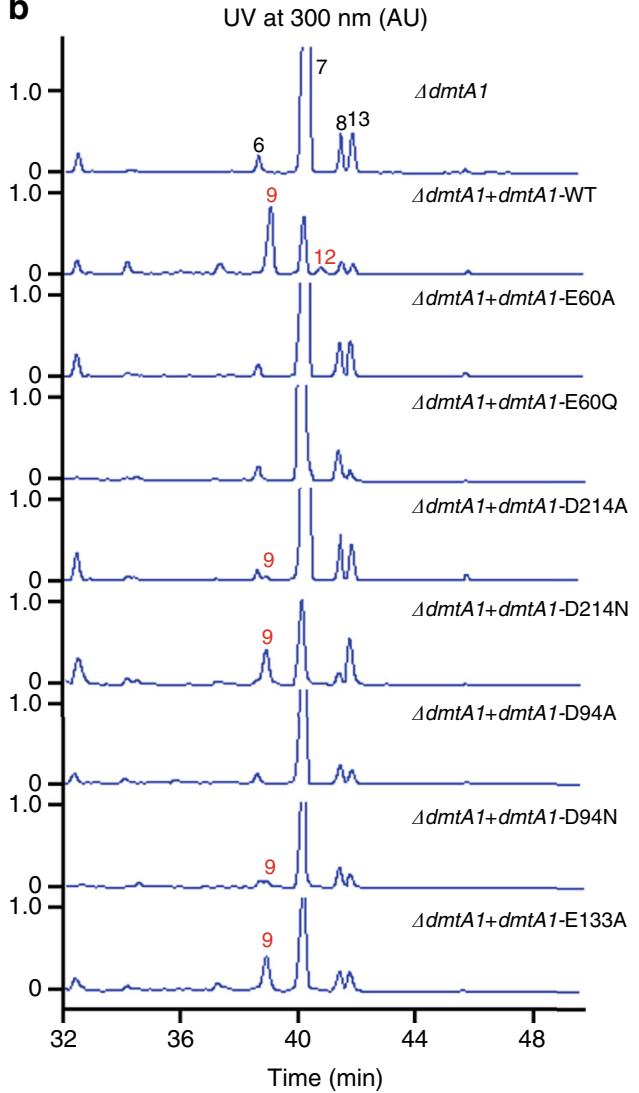

Fig. 8 Impacts of mutagenised DmtA1 on the final cyclization reaction. a Predicted DmtA1 model generated by utilizing TMRPres2D. b HPLC traces of $\Delta d m t A 1$ complemented with different $d m t A 1$ variants (also see Supplementary Fig. 59). The cyclization products $\mathbf{9}$ and $\mathbf{1 2}$ were indicated in red. c Relative formation of DMT G $(\boldsymbol{9})$ production in $\triangle d m t A 1$ complemented with different dmtA1 variants in comparison to complementation with wild-type dmtA1 (WT). Error bars represent \pm s.d. of three independent experiments

L194F (Supplementary Fig. 11) firmly supported this assumption as they led to the production of alternative cWX products. One plausible interpretation is that mutated residues cause an alteration of the binding orientation of the first aa-tRNA substrate and thus directly effect the condensation reactions with the second aatRNA. As the binding pocket $\mathrm{P} 2$ is formed after the binding of the first aa-tRNA to the pocket P1, changes in specificity regarding the second aa-tRNA might alternatively be mediated by a modification of the binding pocket P2. Future crystallographic structure studies might shed light on these underlying molecular mechanisms. It is worth to mention, products formation by CDPSs in vivo is closely related to the relative abundances of specific aa-tRNAs intracellular ${ }^{12,14}$. The in vivo results showed the production of 3 was significantly impaired in DmtB1-L185F and DmtB1-V205M, and thus the major product changed (Fig. 4b); conversely, the in vitro assays indicated both variants still catalyze formation of $\mathbf{3}$ the most but in significantly decreased amount (Fig. 4d). The discrepancy between the in vivo and the in vitro data was also observed for $\mathrm{AlbC}^{14}$. The changes in the ratios of products formation were thus compared (Supplementary Table 7), which clearly showed the consistency between the in vivo and the in vitro data. Taken together, the importance of L185 and V205 for substrate specificity of DmtB1 was unambiguously established.

CDPS genes are often clustered with other tailoring enzymes to generate complex DKP-containing natural products. Modification of the heterocycle or modification of amino acid side chains of the constituent amino acids can therefore be expected. However, so far only four CDPS-dependent assembly lines have been fully elucidated, with modifications such as oxidation ${ }^{16,18,20}$, and methylation $^{18}$. In this study, we were able to demonstrate that $d m t 1$ encodes CDPS-dependent machinery involved in DMT biosynthesis both in vivo and in vitro. DmtB1 produces $c y c l o(\mathrm{~L}-$ Trp-L-Xaa) $(\mathrm{Xaa}=\mathrm{Val}$, Pro, Leu, Ile, and Ala, with cWV being the major product), which undergoes farnesyl transfer followed by cyclization (Fig. 9). Transfer of the farnesyl group is accomplished by a PSL type PT rather than a bacterial indole PT, such as those involved in cyclomarin/cyclomarazine and 6-dimethylallylindole (DMAI)-3-carbaldehyde biosynthesis, CymD ${ }^{33}$ and $\operatorname{IptA}^{34}$, respectively. The only PSL type PT reported so far is NzsG involved in neocarazostatin biosynthesis ${ }^{28}$. NzsG was reported to be highly specific for both the prenyl donor, as well as the prenyl acceptor, transferring dimethylallyl pyrophosphate (DMAPP) onto precarazostatin ${ }^{28}$. In contrast, DmtC1 displays broad substrate specificity regarding to the prenyl acceptor both in vivo (Figs. 5,6) and in vitro (Fig. 7). Our ongoing study reveals that it also shows promiscuity to the prenyl donor. Therefore, $\mathrm{DmtC1}$ could prove to be a powerful biocatalytic tool for accessing structurally diverse compounds.

Noticeably, both the heterologous host and the native producer only produce DMT compound(s) at relatively low titer (Figs. 5, 6). In contrast, pre-DMTs were able to be accumulated in larger amounts (Figs. 5, 6), which indicates that DmtA1 might be rate limiting during the assembly process. Consistently, the yields of DMTs were significantly improved in the $\triangle d m t A 1$ complemented strain (Supplementary Fig. 23). However, in the $d m t A 1$ overexpression strain (in 


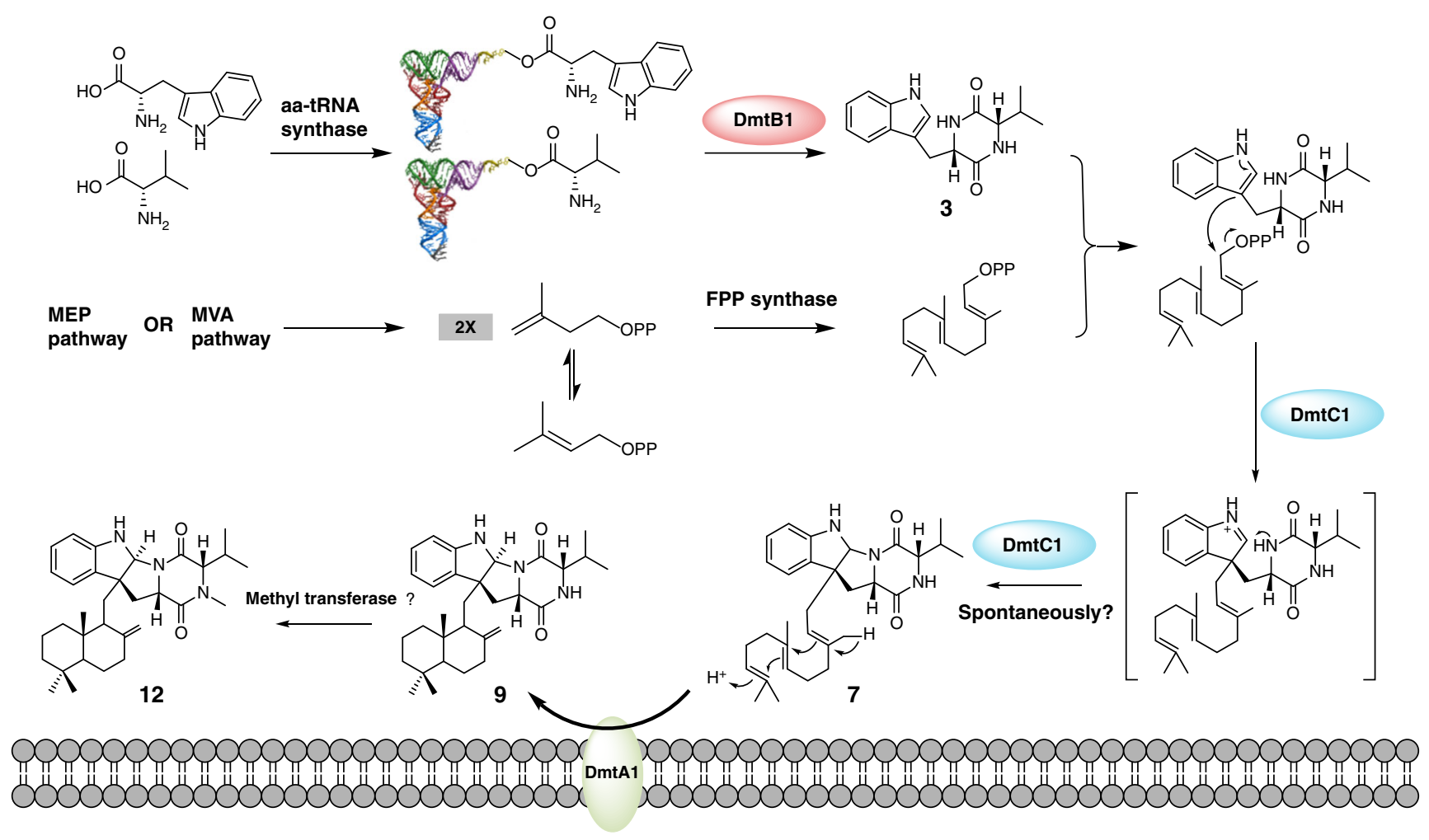

Fig. 9 The CDPS-dependent assembly line of DMT G, 9

the wild-type S. youssoufiensis OUC6819 background), the yields of DMTs were only slightly improved, which might be due to DmtA1 being a membrane protein. As cyclization of pre-DMTs should be initiated by protonation of the C-C double bond (between C-26 and C-27), which is less favored than protonation of the epoxide ring as that happens during xiamycins biosynthesis ${ }^{29,30}$, additional acidic residues may be necessary for catalysis. Our systematic site-directed mutagenesis results demonstrated that, in addition to the conserved E60, the acidic residue D94, unique to DmtA1-3 (absent in XiaE/ $\mathrm{XiaH}$ ), probably plays an important role in catalysis, too (Fig. 8, Supplementary Fig. 58). Very recently, a similar fungal membrane TC MacJ, involved in macrophorins biosynthesis, was identified from Penicillium terrestris, which also bears an additional acidic residue Asp96 related to catalysis ${ }^{35}$. The residue Y33 was also found to be conserved among all the membrane TCs and might contribute to increase the nucleophilicity of the acidic residues. Indeed, substitution of Y33 with either Ala or Phe resulted in inactive DmtA1 variants.

While the wild-type $S$. youssoufiensis OUC6819 is also able to produce DMT F (12) (Fig. 6, Supplementary Fig. 26), which harbors a methyl group at $N-15$ position (Fig. 1), no methyltransferase gene was found adjacent to the $d m t 1$ locus (Supplementary Fig. 12), indicating this gene is probably located in another genomic region. The timing for methylation has still to be investigated. The methyltransferase genes located in the adjacent regions of the $d m t 2 / 3$ loci, as well as the characterized DKP-ring-modifying $N$-methyltransferase Amir_4628 $8^{26}$ would enlighten deciphering of this methylation step. In addition, the presence of the CDO homologs surrounding the $d m t 2 / 3$ loci suggest introduction of $\alpha, \beta$-dehydrogenation on DMTs, thus generating more complex natural products. Significantly, our studies on $d m t 1-3$ have expanded our understanding towards CDPS, as well as CDPS-dependent biosynthetic machineries, opening up new possibilities for combinatorial biosynthesis to enrich the structural diversity of complex DKPs compounds.

\section{Methods}

Materials and general experimental procedures. Bacterial strains and plasmids used and constructed during this study are listed in Supplementary Data 1. S. youssoufiensis OUC6819 (former name: Streptomyces sp. CHQ-64) was isolated from reed rhizosphere soil collected from the mangrove conservation area of Guangdong province, China ${ }^{22}$. Streptomyces sp. NRRL F-5123 and S. aidingensis CGMCC 4.5739 were obtained from Agricultural Research Service Culture Collection (NRRL) and China General Microbiological Collection Center (CGMCC), respectively. The standards of cyclodipeptides were purchased from GL Biochem Ltd (Shanghai, China). Drimentine A, C and G were provided by Prof. Ang Li (Shanghai Institute of Organic Chemist Chemistry, Chinese Academy of Sciences). The prenyl donor farnesyl diphosphate (FPP) was purchased from Sigma-Aldrich. Common biochemicals and chemicals were purchased from standard sources for laboratory use.

All DNA manipulations were performed according to standard procedures ${ }^{36}$ or manufacturer's instruction. Plasmid extractions and DNA purification were carried out using commercial kits (OMEGA, BIO-TEK). Both primer synthesis and DNA sequencing were performed at TSINGKE Biotech Co. Ltd. (Qingdao, China). HRESIMS was carried out on Thermo LTQ Orbitrap XL mass spectrometer. NMR data was recorded with Agilent-DD2-500 or Bruker Avance III 600 spectrometers.

Bioinformatic analysis. ORF assignments and their proposed function were accomplished by using FramePlot4.0 beta $^{37}$ (http://nocardia.nih.go.jp/fp4). Sequence comparisons and database searches were accomplished with BLAST programs ${ }^{38}$ (http://blast.ncbi.nlm.nih.gov/Blast.cgi). The structural model of DmtB1 was generated by using I-TASSER server ${ }^{24}$ (https://zhanglab.ccmb.med. umich.edu/I-TASSER).

Expression of dmt1-3 in E.coli and Streptomyces strains. For the expression of $d m t B 1-3$ in $E$. coli, the responding genes were amplified by polymerase chain reaction (PCR) using primer pairs listed in Supplementary Table 1 from the genomes of $S$. youssoufiensis OUC6819, Streptomyces sp. NRRL F-5123 and S. aidingensis CGMCC 4.5739 , respectively. The amplification products were digested with NdeI and XhoI, ligated into pET-28a resulting in pWLI611-613, which were transformed into E. coli BL21 (DE3) after confirmation by sequencing. For expression of $d m t C 1$ in E. coli, it was amplified from the S. youssoufiensis OUC6819 genome using the primer pair of DmtC1-FP/RP (Supplementary Table 1). The resulting PCR products were digested with EcoRI and XhoI, and ligated into pET-32a to give pWLI627, followed by sequencing and transforming into E. coli BL21 (DE3). 
For the expressions in Streptomyces strains, the corresponding gene(s) was/were put under the control of the constitutive promoter $\mathrm{P}_{\text {gapdh }}$ from S. youssoufiensis OUC6819. Construction was performed as follows: taking $d m t B 1 C 1$ expression plasmid as an example, $\mathrm{P}_{\text {gapdh }}$ was amplified using primer pair of pGFP/pGRP (Supplementary Table 2) and was digested with NsiI; the dmtB1C1 fragment was amplified with the primer pair of dmtB1C1-FP/dmtC1-RP (Supplementary Table 2) and digested with $\mathrm{XbaI}$; and then these two digested fragments were ligated and cloned into the NsiI and XbaI sites of pSET152C to give pWLI615. Similarly, the fragments of $d m t A 1 B 1 C 1, d m t B 2 C 2, d m t A 2 B 2 C 2, d m t B 3 C 3$, and $d m t A 3 B 3 C 3$ were cloned into the same sites of pSET152C to obtain pWLI616-620 (Supplementary Data 1), respectively. After confirmation by sequencing, the resulting plasmids were passed through E. coli ET12567/pUZ8002 and then introduced into S. coelicolor M1146 via conjugation ${ }^{39}$.

Construction of site-directed mutagenesis variants. Site-directed mutagenesis was accomplished by using SOE-PCR method ${ }^{40}$. All PCR primers are listed in Supplementary Table 3 (for DmtB1 and DmtB2 variants construction) and Supplementary Table 4 (for DmtA1 variants construction). Taking the variant L185F as an example, the recombinant plasmid pWLI611 was used as template in the first and second PCR reactions, using the primer pairs of DmtB1-FP/DmtB1L185F-RP and DmtB1-RP/DmtB1L185F-FP. The resulting PCR products were purified and served as a template for the third round of PCR using the primer pair of DmtB1FP/DmtB1-RP. After digestion with NdeI and XhoI, the amplified products were ligated into pET-28a and transformed into E. coli $\mathrm{DH} 5 \alpha$. The final recombinant plasmid was sequenced to ensure the accuracy of the intended gene mutation and then transformed into E. coli BL21 (DE3). For the purpose of probing the key residues in DmtAl, the resulting variants were introduced into $\triangle d m t A 1$ via intergenic conjugation.

Identification of DKP compounds formed by DmtB1-3. Overnight cultures of $E$. coli BL21 (DE3) cells containing expression constructs of DmtB1-3 were used to inoculate $[1: 50(\mathrm{v} / \mathrm{v})]$ into $50 \mathrm{~mL}$ of M9 minimal medium ${ }^{26}$ containing $50 \mu \mathrm{g} \mathrm{mL} \mathrm{L}^{-1}$ kanamycin. Expression of the recombinant proteins was induced at an $\mathrm{OD}_{600}$ of approximately 0.8 by addition of isopropyl- $\beta$ - $D$ - 1 -thiogalactopyranoside (IPTG, $0.1 \mathrm{mM}$ final concentration), and cultivation was continued for additional $20 \mathrm{~h}$ at $16^{\circ} \mathrm{C}$. The cells were collected by centrifuging at $10,000 \times g$ for $20 \mathrm{~min}$, resuspended in $2 \mathrm{~mL}$ of ice-cold binding buffer $(0.05 \mathrm{M}$ Tris- $\mathrm{HCl}, 0.5 \mathrm{M} \mathrm{NaCl}, \mathrm{pH} 7.5$, containing cOmplete ${ }^{\mathrm{TM}}$ protease inhibitor cocktail) and lysed by sonication at the same time. Proteins in crude extracts were detected by western blotting according to manufacturer's instruction. The $\mathrm{His}_{6}{ }$-tagged proteins were detected with primary antibody (Cell Signaling Technology: CST, \# 2365 at 1:1000) and visualized using SuperSignal ${ }^{\text {mo }}$ West Femto Substrate Trial Kit (Thermo Scientific, \# 34094)

The supernatants of the cultures were extracted three times with an equal volume of ethyl acetate. The combined extracts were concentrated in vacuo and dissolved in $1 \mathrm{~mL}$ of MeOH. HPLC analyses were conducted using an Agilent 1260 HPLC system equipped with a YMC-Pack ODS-AQ C18 column $(150 \mathrm{~mm} \times 4.6$ $\mathrm{mm}$, particle size of $5 \mu \mathrm{m}$, pore size of $120 \AA$ ) under the following program: solvent A consisting of $0.1 \%(\mathrm{v} / \mathrm{v})$ formic acid and $\mathrm{ddH}_{2} \mathrm{O}$, solvent $\mathrm{B}$ consisting of $0.1 \%(\mathrm{v} /$ v) formic acid and acetonitrile; $10 \% \mathrm{~B}(0-5 \mathrm{~min}), 10 \%$ to $50 \% \mathrm{~B}(5-25 \mathrm{~min}), 100 \%$

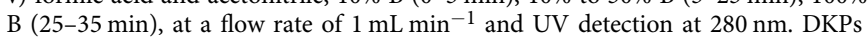
were quantified on the basis of their peak area at $280 \mathrm{~nm}$ using calibration curves obtained with authentic standards.

\section{Genomic library construction and library screening. Genomic DNA of $S$.} youssoufiensis OUC6819 was partially digested with Sau3AI, and fragments with the size of $40-50 \mathrm{~kb}$ were recovered and dephosphorylated with FastAP (Thermo Scientific, Pittsburgh, USA), and then ligated into SuperCos1 that was pretreated with $X b a I$, dephosphorylated, and digested with BamHI. The ligation product was packaged into lambda particles with the MaxPlax Lambda Packaging Extract (Epicenter, Madison, WI, USA) as per the manufacture's instruction and plated on E. coli Top 10 . The titer of the primary library was about $5 \times 10^{6} \mathrm{cfu}$ per $\mu \mathrm{g}$ of DNA. The primer pair used for cosmid library screening is listed in Supplementary Table 5.

Gene manipulation. Gene inactivation in S. youssoufiensis OUC6819 was performed using the REDIRECT Technology according to the literature protocol ${ }^{41}$. The aac(3)IV-oriT resistance cassette was amplified with appropriate primers (Supplementary Table 6) using pIJ773 as template and was transformed into E. coli BW25113/pIJ790 containing cosmid pWLI614 to replace an internal region of $d m t A 1, d m t B 1$ and $d m t C 1$, respectively, resulting in mutant cosmids pWLI621-623, respectively. pWLI621-623 were passed through E. coli ET12567/pUZ8002 and then introduced into $S$. youssoufiensis OUC6819 by intergenic conjugation using mycelia as recipients. A 24-h-old $5 \mathrm{~mL}$ of $S$. youssoufiensis OUC6819 culture in RARE3 medium $\left(10 \mathrm{~g} \mathrm{~L}^{-1}\right.$ glucose, $4 \mathrm{~g} \mathrm{~L}^{-1}$ yeast extract, $10 \mathrm{~g} \mathrm{~L}^{-1}$ malt extract, $2 \mathrm{~g} \mathrm{~L}$

${ }^{-1}$ Bacto peptone, $2 \mathrm{~g} \mathrm{~L}^{-1} \mathrm{MgCl}_{2} \cdot \mathrm{H}_{2} \mathrm{O}, 5 \mathrm{~g} \mathrm{~L}^{-1}$ glycerol, $5 \mathrm{~g} \mathrm{~L}^{-1}$ glycine) was diluted $1: 10$ followed by additional incubation at $30^{\circ} \mathrm{C}$ for $24 \mathrm{~h}$. The mycelia were then collected by centrifugation and resuspended in equal volume of RARE3 medium, and were homogenized and fragmented by sonication with an ultrasonic processors VCX750 (Sonics and Materials Inc, PA, USA). After incubation at $30^{\circ} \mathrm{C}$ for another $18 \mathrm{~h}$, the mycelia were treated again as described above, resulting in recipient cells. The donor cells, E. coli ET12567/pUZ8002 carrying the desired conjugative plasmid, and the recipient cells were mixed in 1:10 ratio and plated on ISP4 ${ }^{39}$ agar supplemented with $20 \mathrm{mM} \mathrm{MgCl}_{2}$. The desired mutants were selected by the apramycin-resistant and kanamycin-sensitive phenotype, and were further confirmed by PCR (Supplementary Table 6, Supplementary Figs. 20-22). For genetic complementation and overexpression experiments, the $d m t A 1, d m t B 1$, and $d m t C 1$ expression plasmids were constructed in a similar way as that for the $d m t B 1 C 1$ expression plasmid, generating pWLI624-626, respectively. pWLI624626 were passed through E. coli ET12567/pUZ8002 and then introduced into the wild-type and mutant strains for gene overexpression and genetic complementation, respectively, via intergenic conjugation as described above.

Production and analysis of fermentation products. Streptomyces strains, including heterologous expression strains, mutant strains, genetic complementary strains, and overexpression strains, were incubated on a rotatory shaker at $30^{\circ} \mathrm{C}$ in $250 \mathrm{~mL}$ Erlenmeyer flasks each containing $50 \mathrm{~mL}$ of fermentation medium composed of $1 \%$ soluble starch, $2 \%$ glucose, $4 \%$ corn syrup, $1 \%$ yeast extract, $0.3 \%$ beef extract, $0.05 \% \mathrm{MgSO}_{4} \cdot 7 \mathrm{H}_{2} \mathrm{O}, 0.05 \% \mathrm{KH}_{2} \mathrm{PO}_{4}, 0.2 \% \mathrm{CaCO}_{3}$, and $3 \%$ sea salt, $\mathrm{pH} 7.0$. After 7 days of cultivation, the fermentation cultures were harvested by centrifugation. The supernatants were extracted twice with an equal volume of ethyl acetate, and the combined EtOAc extracts were concentrated in vacuo to afford residue $\mathrm{A}$. The precipitated mycelia were extracted twice with acetone. The extracts were combined, and acetone was evaporated in vacuo to yield residue $B$. The combined residues were dissolved in methanol and filtered through a $0.2 \mu \mathrm{m}$ filter. The resulting fermentation products were detected by HPLC analysis, using a YMC-Pack ODS-AQ C18 column with UV detection at $300 \mathrm{~nm}$ under the following program: solvent (phase $\mathrm{A}, 0.1 \%$ formic acid in $\mathrm{ddH}_{2} \mathrm{O}$; phase $\mathrm{B}, 100 \%$ acetonitrile supplemented with $0.1 \%$ formic acid); $20 \%$ B ( $0-5 \mathrm{~min}), 20 \%$ to $100 \%$ B (5-45 min), $100 \%$ B (45-55 min), at a flow rate of $1 \mathrm{~mL} \mathrm{~min}^{-1}$.

Isolation of pre-drimentines and drimentines. The $\triangle d m t A 1$ mutant strain and $S$. coelicolor M1146 expressing dmtA1B1C1 or $d m t A 2 B 2 C 2$ were fermented in volume of $20 \mathrm{~L}$ to isolate compounds 6-8 and $\mathbf{1 3}$ (from $\Delta d m t A 1$ ), $\mathbf{9}$ (from M1146/ pWLI616), 10 and 11 (from M1146/pWLI618), respectively. The fermentation cultures were treated as described above. The residues were applied to reversedphase $\mathrm{C} 18$ open column, eluting with a gradient eluent of $20 \%-100 \%$ methanol to give five fractions (Fr.1 Fr.5) for each fermentation culture. Compounds 6-8 and 13 were obtained by further separation of the Fr.4 from $\triangle d m t A 1$, eluting with linear gradient from 80 to $100 \% \mathrm{~B} / \mathrm{A}$ (phase $\mathrm{A}$ : $\mathrm{ddH}_{2} \mathrm{O}$; phase $\mathrm{B}$ : acetonitrile, $2 \mathrm{~mL}$ $\mathrm{min}^{-1}$, UV detection at $300 \mathrm{~nm}$ ) in $40 \mathrm{~min}$ using a semi-preparative HPLC column (YMC-Pack ODS-AA C18 column, $120 \AA$, $250 \times 10 \mathrm{~mm}, 5 \mu \mathrm{m}$ ). Compounds 9-11 were also obtained by separation of the Fr.4 from M1146/pWLI616 (for 9) and M1146/pWLI618 (for 10-11) under the same HPLC conditions. The structures of the compounds were characterized by HR-ESIMS and NMR spectroscopy. All NMR spectra were processed with MestReNova.6.1.0 (Metrelab), and chemical shifts were referenced to those of the solvent DMSO- $d_{6}$ signals.

Protein expression and purification. $10 \mathrm{~mL}$ overnight culture of E. coli BL21 (DE3) harboring DmtB1 or DmtC1 expression plasmid was inoculated into $1 \mathrm{~L}$ of LB medium (containing $50 \mu \mathrm{g} \mathrm{mL}-1$ kanamycin or ampicillin) and grown at $37^{\circ} \mathrm{C}$ with shaking. Expression was induced at an $\mathrm{OD}_{600}$ of approximately 0.6 by addition of IPTG (0.1 and $0.05 \mathrm{mM}$ final concentration for DmtB1 and DmtC1, respectively), and cultivation was continued for additional $16 \mathrm{hrs}$ at $16^{\circ} \mathrm{C}$.

The cells were pelleted by centrifugation $(15 \mathrm{~min}$ at $8000 \times \mathrm{g})$ and resuspended in $30 \mathrm{~mL}$ of binding buffer A $(0.15 \mathrm{M} \mathrm{NaCl}, 5.0 \%$ glycerol $(\mathrm{v} / \mathrm{v}), 1.0 \%(\mathrm{v} / \mathrm{v})$ Triton X100, $0.1 \mathrm{M}$ Tris- $\mathrm{HCl}, \mathrm{pH} 8.0$, containing cOmplete ${ }^{\mathrm{TM}}$ protease inhibitor cocktail) for DmtB1 expression and $(0.05 \mathrm{M}$ Tris- $\mathrm{HCl}, 0.5 \mathrm{M} \mathrm{NaCl}, \mathrm{pH} 7.5$, containing cOmplete $^{\mathrm{TM}}$ protease inhibitor cocktail) for DmtC1 expression. The resuspended cells were lysed by sonication in an ice-water bath with an ultrasonic processors VCX750 (Sonics and Materials Inc, PA, USA), and centrifuged at 10,000 $\times g$ for 30 min at $4{ }^{\circ} \mathrm{C}$. The supernatant was applied to a HisTrap HP column $(1 \mathrm{~mL}, \mathrm{GE}$ Healthcare) and the His-tagged DmtC1 protein was eluted with a linear gradient of imidazole (30-500 $\mathrm{mM})$ in the binding buffer using an ÄKTA Purifier system. After SDS-PAGE analysis, fractions containing pure DmtC1 were pooled, concentrated and exchanged to Tris buffer $(0.025 \mathrm{M}$ Tris- $\mathrm{HCl}, 0.02 \mathrm{M} \mathrm{NaCl}$, and $10.0 \%$ glycerol, $\mathrm{pH} 7.5$ ) by using Amicon Ultra- $1530-\mathrm{kDa}$ cutoff centrifugal concentrator (Millipore). And in case of DmtB1 and its variants, proteins were treated with RNase A and further subjected to a Heparin HP column $(1 \mathrm{~mL}, \mathrm{GE}$ Healthcare). Proteins were eluted with a linear gradient from $100 \%$ buffer B $(0.1 \mathrm{M}$ Tris- $\mathrm{HCl} \mathrm{pH} 8.0,0.05 \mathrm{M} \mathrm{NaCl})$ to $100 \%$ buffer C $(0.1 \mathrm{M}$ Tris- $\mathrm{HCl}, \mathrm{pH} 8.0,1.0 \mathrm{M}$ $\mathrm{NaCl}$ ) over $30 \mathrm{~min}$ at a flow rate of $0.5 \mathrm{~mL} \mathrm{~min}^{-1}$. Fractions containing pure proteins, as evidenced by SDS-PAGE, were concentrated and the buffer was exchanged to buffer D (0.1 M Tris- $\mathrm{HCl}, \mathrm{pH} 8.0,0.15 \mathrm{M} \mathrm{NaCl}, 5.0 \%$ glycerol (v/v) and $10 \mathrm{mM}$ beta-mercaptoethanol) using a Amicon Ultra-15 10-kDa cutoff centrifugal concentrator (Millipore).

assays. A typical assay for DmtB1 and its variants was completed using established methods ${ }^{18}$. Briefly, recombinant DmtB1 or its variants $(15 \mu \mathrm{M})$ was incubated with 
$50 \mu \mathrm{M}$ tRNA mix from E.coli (Sigma, \# R1753), aminoacyl-tRNA synthetase from E.coli (250 U, Sigma, \# A3646), $5 \mathrm{mM}$ amino acids (L-Trp, L-Val, L-Pro, L-Leu, and L-Ile), $5 \mathrm{mM}$ ATP, $10 \mathrm{mM} \mathrm{MgCl}$, $30 \mathrm{mM} \mathrm{KCl,} 100 \mathrm{mM}$ Tris-HCl buffer (pH 8.0) and RNase inhibitor (20 U, Roche) were incubated in a total volume of $100 \mu \mathrm{L}$ for $20 \mathrm{~h}$ at $30^{\circ} \mathrm{C}$. Reactions were quenched with TFA $(2 \mu \mathrm{L}, 2 \% \mathrm{v} / \mathrm{v})$, centrifuged $(17,000 \times g)$ for $30 \mathrm{~min}$ and the supernatants were subjected to LC-MS ${ }^{2}$. Assays were conducted in duplicates.

The Agilent series 1290 HPLC system equipped with Agilent 6430 triple quadrupole mass spectrometry was used for the LC-MS ${ }^{2}$ analysis. All separations were carried out on a reversed-phase Thermos Hypersil GOLD C18 column (100 $\mathrm{mm} \times 2.1 \mathrm{~mm}, 1.9 \mu \mathrm{m}, 175 \AA$, Thermo Scientific Inc., USA) under the following program: solvent A consisting of $0.1 \%(\mathrm{v} / \mathrm{v})$ formic acid and $\mathrm{dd}_{2} \mathrm{O}$, solvent $\mathrm{B}$ consisting of $0.1 \%(\mathrm{v} / \mathrm{v})$ formic acid and acetonitrile; $10 \% \mathrm{~B}(0-5 \mathrm{~min}), 10 \%$ to $50 \%$ B (5-30 min), 100\% B (30-40 min), at a flow rate of $0.2 \mathrm{~mL} \mathrm{~min}^{-1}$. The optimized mass spectrometric parameters including fragmentor voltage (FG), collision energy (CE), and multiple reaction monitoring (MRM) transitions for each compound are shown in Supplementary Table 8. Agilent Mass Hunter software rev. B.03.01 was used for analysis.

The enzymatic assay of DmtCl was carried out in $50 \mathrm{mM}$ Tris- $\mathrm{HCl}$ buffer $(\mathrm{pH}$ 8.0) with $2.5 \mathrm{mM} \mathrm{MgCl}_{2}$, containing $10 \mu \mathrm{M} \mathrm{DmtCl}, 1 \mathrm{mM}$ DKP, and $0.2 \mathrm{mM} \mathrm{FPP}$. The optimal assay conditions were obtained at $30^{\circ} \mathrm{C}$. After $1 \mathrm{hr}$, the reaction was quenched by the addition of equal volume of methanol and mixed by vortexing. The mixture was centrifuged at $17,000 \times g$ for $20 \mathrm{~min}$ to remove protein. The supernatant was then subjected to HPLC analysis under the identical conditions used for analyzing the fermentation products of the Streptomyces strains.

\section{Data availability}

The nucleotide sequences of the $d m t 1$ locus, its upstream gene orf $(-1)$ and downstream genes orf1 and orf 2 have been deposited in the GenBank database under accession numbers of MG776357 [https://www.ncbi.nlm.nih.gov/nuccore/MG776357], MH666061 [https://www.ncbi.nlm.nih.gov/nuccore/MH666061], MH666062 [https://www.ncbi.nlm. nih.gov/nuccore/MH666062] and MH666063 [https://www.ncbi.nlm.nih.gov/nuccore/ MH666063], respectively. All relevant data supporting the findings of this study are available within the paper and its supplementary information files. Additional data are upon reasonable request.

Received: 25 February 2018 Accepted: 30 August 2018

Published online: 05 October 2018

\section{References}

1. Borthwick, A. D. 2,5-Diketopiperazines: synthesis, reactions, medicinal chemistry, and bioactive natural products. Chem. Rev. 112, 3641-3716 (2012).

2. Teixidó, M., Zurita, E., Malakoutikhah, M., Tarragó, T. \& Giralt, E. Diketopiperazines as a tool for the study of transport across the blood-brain barrier (BBB) and their potential use as BBB-shuttles. J. Am. Chem. Soc. 129, 11802-11813 (2007).

3. Nicholson, B. et al. NPI-2358 is a tubulin-depolymerizing agent: in-vitro evidence for activity as a tumor vascular-disrupting agent. Anticancer Drugs 17, 25-31 (2006)

4. Kanoh, K. et al. Antitumor activity of phenylahistin in vitro and in vivo. Biosci. Biotechnol. Biochem. 63, 1130-1133 (1999).

5. Miyoshi, T., Miyairi, N., Aoki, H., Kosaka, M. \& Sakai, H. Bicyclomycin, a new antibiotic. J. Antibiot. 25, 569-575 (1972).

6. Walsh, C. T. Insights into the chemical logic and enzymatic machinery of NRPS assembly lines. Nat. Prod. Rep. 33, 127-135 (2016).

7. Gondry, M. et al. Cyclodipeptide synthases are a family of tRNA-dependent peptide bond-forming enzymes. Nat. Chem. Biol. 5, 414-420 (2009).

8. Belin, P. et al. The nonribosomal synthesis of diketopiperazines in tRNAdependent cyclodipeptide synthase pathways. Nat. Prod. Rep. 29, 961-979 (2012).

9. Giessen, T. W. \& Marahiel, M. A. The tRNA-dependent biosynthesis of modified cyclic dipeptides. Int. J. Mol. Sci. 15, 14610-14631 (2014).

10. Gondry, M. et al. A comprehensive overview of the cyclodipeptide synthase family enriched with the characterization of 32 new enzymes. Front. Microbiol. 9, 46 (2018)

11. Jacques, I. B. et al. Analysis of 51 cyclodipeptide synthases reveals the basis for substrate specificity. Nat. Chem. Biol. 11, 721-727 (2015).

12. Moutiez, M., Belin, P. \& Gondry, M. Aminoacyl-tRNA-utilizing enzymes in natural product biosynthesis. Chem. Rev. 117, 5578-5618 (2017).

13. Canu, N. et al. Incorporation of non-canonical amino acids into 2,5diketopiperazines by cyclodipeptide synthases. Angew. Chem. 130, 3172-3176 (2018).
14. Moutiez, M. et al. Specificity determinants for the two tRNA substrates of the cyclodipeptide synthase AlbC from Streptomyces noursei. Nucleic Acids Res. 42, 7247-7258 (2014).

15. Skinnider, M. A., Johnston, C. W., Merwin, N. J., Dejong, C. A. \& Magarvey, N. A. Global analysis of prokaryotic tRNA-derived cyclodipeptide biosynthesis. BMC Genom. 19, 45 (2018).

16. Lautru, S., Gondry, M., Genet, R. \& Pernodet, J. L. The albonoursin gene Cluster of $S$. noursei: biosynthesis of diketopiperazine metabolites independent of nonribosomal peptide synthetases. Chem. Biol. 9, 1355-1364 (2002).

17. Fu, P. et al. a-Pyrones and diketopiperazine derivatives from the marinederived actinomycete Nocardiopsis dassonvillei HR10-5. J. Nat. Prod. 74, 2219-2223 (2011)

18. Giessen, T. W., von Tesmar, A. M. \& Marahiel, M. A. Insights into the generation of structural diversity in a tRNA-dependent pathway for highly modified bioactive cyclic dipeptides. Chem. Biol. 20, 828-838 (2013).

19. Cryle, M. J., Bell, S. G. \& Schlichting, I. Structural and biochemical characterization of the cytochrome P450 CypX (CYP134A1) from Bacillus subtilis: a cyclo-L-leucyl-L-leucyl dipeptide oxidase. Biochemistry 49, 7282-7296 (2010)

20. Meng, S. et al. A six-oxidase cascade for tandem C-H bond activation revealed by reconstitution of bicyclomycin biosynthesis. Angew. Chem. Int. Ed. Engl. 57, 719-723 (2018).

21. Patteson, J. B., Cai, W., Johnson, R. A., Santa Maria, K. C. \& Li, B. Identification of the biosynthetic pathway for the antibiotic bicyclomycin. Biochemistry 57, 61-65 (2018).

22. Che, Q. et al. Hybrid isoprenoids from a reeds rhizosphere soil derived actinomycete Streptomyces sp. CHQ-64. Org. Lett. 14, 3438-3441 (2012)

23. Liu, J., Yu, H. L. \& Li, S. M. Expanding tryptophan-containing cyclodipeptide synthase spectrum by identification of nine members from Streptomyces strains. Appl. Microbiol. Biotechnol. 102, 4435-4444 (2018).

24. Roy, A., Kucukural, A. \& Zhang, Y. I-TASSER: a unified platform for automated protein structure and function prediction. Nat. Protoc. 5, 725 (2010).

25. Bonnefond, L. et al. Structural basis for nonribosomal peptide synthesis by an aminoacyl-tRNA synthetase paralog. Proc. Natl Acad. Sci. USA 108, 3912-3917 (2011)

26. Giessen, T. W., von Tesmar, A. M. \& Marahiel, M. A. A tRNA-dependent two enzyme pathway for the generation of singly and doubly methylated ditryptophan 2,5-diketopiperazines. Biochemistry 52, 4274-4283 (2013).

27. Skiredj, A., Beniddir, M. A., Evanno, L. \& Poupon, E. Mimicking the main events of the biosynthesis of Drimentines: synthesis of $\Delta^{8 \prime}$-isodrimentine A and related compounds. Eur. J. Org. Chem. 2016, 2954-2958 (2016)

28. Huang, S. et al. Biosynthesis of neocarazostatin A reveals the sequential carbazole prenylation and hydroxylation in the tailoring steps. Chem. Biol. 22, 1633-1642 (2015)

29. Xu, Z., Baunach, M., Ding, L. \& Hertweck, C. Bacterial synthesis of diverse indole terpene alkaloids by an unparalleled cyclization sequence. Angew. Chem. Int. Ed. Engl. 51, 10293-10297 (2012).

30. $\mathrm{Li}, \mathrm{H}$. et al. Elucidating the cyclization cascades in xiamycin biosynthesis by substrate synthesis and enzyme characterizations. Org. Lett. 17, 306-309 (2014).

31. Itoh, T. et al. Reconstitution of a fungal meroterpenoid biosynthesis reveals the involvement of a novel family of terpene cyclases. Nat. Chem. 2, 858-864 (2010).

32. Thoma, R. et al. Insight into steroid scaffold formation from the structure of human oxidosqualene cyclase. Nature 432, 118-122 (2004).

33. Schultz, A. W., Lewis, C. A., Luzung, M. R., Baran, P. S. \& Moore, B. S. Functional characterization of the cyclomarin/cyclomarazine crenyltransferase CymD directs the biosynthesis of unnatural cyclic peptides. J. Nat. Prod. 73, 373-377 (2010)

34. Takahashi, S. et al. Biochemical characterization of a novel indole prenyltransferase from Streptomyces sp. SN-593. J. Bacteriol. 192, 2839-2851 (2010)

35. Tang, M. C. et al. Late-stage terpene cyclization by an integral membrane cyclase in the biosynthesis of isoprenoid epoxycyclohexenone natural products. Org. Lett. 19, 5376-5379 (2017).

36. Sambrook, J. \& Russell, D. W. in Molecular Cloning: A Laboratory Manual. Third. (Cold pring Harbor Laboratory Press, New York, 2001).

37. Ishikawa, J. \& Hotta, K. FramePlot: a new implementation of the frame analysis for predicting protein-coding regions in bacterial DNA with a high G + C content. FEMS Microbiol. Lett. 174, 251-253 (1999).

38. McGinnis, S. \& Madden, T. L. BLAST: at the core of a powerful and diverse set of sequence analysis tools. Nucleic Acids Res. 32, W20-W25 (2004).

39. Kieser, T. in Practical Streptomyces Genetics. (John Innes Foundation, Norwich, United Kingdom, 2000).

40. Heckman, K. L. \& Pease, L. R. Gene splicing and mutagenesis by PCR-driven overlap extension. Nat. Protoc. 2, 924-932 (2007).

41. Gust, B., Kieser, T. \& Chater, K. in REDIRECT Technology: Pcr-targeting System in Streptomyces Coelicolor. (The John Innes Centre, Norwich, United Kingdom, 2002). 


\section{Acknowledgements}

We would like to thank Prof. Christopher Corre from the University of Warwick and Prof. Liangcheng Du from the University of Nebraska-Lincoln for constructive suggestions and discussions, Prof. Ang Li from Shanghai Institute of Organic Chemist Chemistry, CAS, for kindly providing us DMT standards, Prof. Mervyn J. Bibb (John Innes Centre, UK) for kindly providing us S. coelicolor M1146, and the ARS Culture Collection (NRRL) for kindly providing Streptomyces sp. NRRL F-5123. This work was supported by grants from the National Natural Science Foundation of China $(31171201,31570032,31711530219,41506157$, and 81561148012), and the NSFC-Shandong Joint Foundation (U1706206 and U1406403).

\section{Author contributions}

T.Y. and J.L. performed all the experiments. Z.L. and T.L. assisted in genetic manipulation of S. youssoufiensis OUC6819 and bioinformatics analysis. H.L. was involved in NMR analysis. Q.C., T.Z., D.L., and Q.G. isolated the S. youssoufiensis OUC6819 strain and identified DMTG and F.T.Y. was involved in the draft manuscript writing. WL supervised the whole work and wrote the manuscript. All authors read and approved the final manuscript.

\section{Additional information}

Supplementary Information accompanies this paper at https://doi.org/10.1038/s41467018-06411-x.
Competing interests: The authors declare no competing interests.

Reprints and permission information is available online at http://npg.nature.com/ reprintsandpermissions/

Publisher's note: Springer Nature remains neutral with regard to jurisdictional claims in published maps and institutional affiliations.

Open Access This article is licensed under a Creative Commons Attribution 4.0 International License, which permits use, sharing, adaptation, distribution and reproduction in any medium or format, as long as you give appropriate credit to the original author(s) and the source, provide a link to the Creative Commons license, and indicate if changes were made. The images or other third party material in this article are included in the article's Creative Commons license, unless indicated otherwise in a credit line to the material. If material is not included in the article's Creative Commons license and your intended use is not permitted by statutory regulation or exceeds the permitted use, you will need to obtain permission directly from the copyright holder. To view a copy of this license, visit http://creativecommons.org/ licenses/by/4.0/.

(C) The Author(s) 2018 